\title{
¿Cómo evaluar los aprendizajes en matemáticas?
}

\section{How to evaluate learning in mathematics?}

César Augusto Trelles Zambrano

Universidad de Cuenca, Ecuador

Fabián Eugenio Bravo Guerrero

Universidad de Cuenca, Ecuador

Juan Fernando Barrazueta Samaniego

Universidad de Cuenca, Ecuador

Autor por Correspondencia: cesar.trellesz@ucuenca.edu.ec, fabian.bravo@ucuenca.edu.ec, juan.barrazueta@ucuenca.edu.ec

Fecha de recepción: 13 de Marzo de 2017 - Fecha de aceptación: 5 de Junio de 2017

\section{Resumen}

El presente documento hace un abordaje teórico de lo que implica la evaluación de los aprendizajes, analiza las etapas de un proceso de evaluación, así como las funciones y tipos de evaluación que se presentan en el proceso educativo. Finalmente considerando que el Sistema Nacional de Educación ha experimentado una serie de cambios en su currículo en los últimos años se elabora una propuesta de las diferentes técnicas de evaluación con sus respectivos instrumentos que pueden ser aplicados en la evaluación de los aprendizajes en la asignatura de Matemáticas.

Palabras clave: evaluación de los aprendizajes; técnicas de evaluación; instrumentos de evaluación; educación matemática

\begin{abstract}
This present paper makes a theoretical approach which involves the learning evaluation, analyzes the stages of its procedure as well as the functions and the types of assessment in all the educational process. Additionally, it has taken into consideration the series of changes in the curriculum in recent years and has prepared a proposal of different assessment techniques with their corresponding instruments. These ones could be applied to assess the learning in Mathematics.
\end{abstract}

Key words: learning assessment; assessment techniques; assessment instruments; evaluation; mathematics education 


\section{Introducción}

Muchos son los cambios que se han implementado en el Sistema Nacional de Educación durante los últimos años, pasando por la aprobación de la Ley Orgánica de Educación Intercultural y su Reglamento, la Actualización y Fortalecimiento Curricular de la Educación General Básica, la puesta en vigencia del Bachillerato General unificado y el último reajuste curricular elaborado en 2016.

En todos los documentos mencionados se manifiesta el objetivo de elevar la calidad de la educación de nuestro sistema educativo. Como parte de este proceso, el Ministerio de Educación, mediante sus documentos curriculares ha estandarizado los objetivos educativos para todos los niveles de Educación General Básica y Bachillerato General Unificado, así como mediante la formulación de las orientaciones metodológicas, ha puesto a consideración la metodología que debe ser adoptada por los docentes en el ánimo de conseguir aquellos objetivos.

Sin embargo consideramos que estos cambios deben ir a la par, con una profunda reflexión del profesorado en torno a su práctica docente, y de forma particular respecto a la evaluación de los aprendizajes. "Si las prácticas de evaluación no se modifican, los supuestos cambios pedagógicos en la enseñanza no tendrán ninguna repercusión ni en el sistema educativo ni en el alumnado" (Díaz Barriga \& Hernández, 2002).

Es por ello que el presente documento pretende aportar con algunos aspectos teóricos relacionados al ámbito de la evaluación que sirvan como punto de partida para ese proceso de reflexión tan importante en los actuales momentos, se abordarán también aspectos prácticos sobre el tema en cuestión, enfatizando que la idea no es la de dotar de una receta a ser aplicada por los docentes, sino más bien aportar con una propuesta que permita abrir una amplia gama de posibilidades para ser trabajadas en las aulas.

\section{Construcción del Concepto de Evaluación}

La actividad evaluativa es inherente a los procesos educacionales, empero vale cuestionarnos sobre ¿cómo entendemos este proceso?, ¿cómo lo llevamos a la práctica en nuestro accionar diario?, ¿evaluamos porque el sistema nos lo exige? o ¿evaluamos para mejorar continuamente el proceso educativo?, ¿evaluamos apegados a un marco teórico conceptual? o ¿entendemos la evaluación como el simple hecho de tomar pruebas y asignar calificaciones?, ¿evaluamos permanentemente el aprendizaje de nuestros estudiantes? o ¿evaluamos simplemente al término de una unidad, bloque, capítulo o ciclo académico?, las respuestas que los docentes den a las anteriores preguntas, sin duda reflejan la concepción que tienen sobre educación. Según Quinquer (1999) la forma en cómo se aborda la evaluación de los aprendizajes tiene relación directa con las concepciones que tienen los docentes sobre la enseñanza y el aprendizaje.

A continuación exponemos algunas pautas que permitan aproximarnos al concepto como paso previo para adentrarnos en lo que significa evaluar en la escuela.

Muchas de las decisiones que las personas toman en el día a día están supeditadas a procesos evaluativos de distinta índole, por ejemplo si una persona piensa adquirir un vehículo y 
tiene diferentes opciones, realiza una evaluación de las mismas para decidirse por la mejor. Si una persona debe realizar un viaje, realiza una evaluación de cuál es la mejor ruta para llegar a su destino, etc. De manera similar en el proceso educativo, se puede evaluar los aprendizajes de los estudiantes, el desempeño del docente, la infraestructura educativa, las políticas educativas, etc., el objetivo que debe primar en todos estos procesos es tomar las mejores decisiones que permitan conseguir los objetivos educacionales.

La evaluación aplicada a la enseñanza y al aprendizaje consiste en un proceso sistemático y riguroso de recogida de datos, incorporado al proceso educativo desde su comienzo, de manera que sea posible disponer de información continua y significativa para conocer la situación, formar juicios de valor con respecto a ella y toma de decisiones adecuadas para proseguir la actividad educativa mejorándola progresivamente. (Casanova, 1998).

Para otros autores, la evaluación educativa es "aquel proceso orientado hacia la determinación, búsqueda y obtención de evidencias acerca del grado y nivel de calidad del aprendizaje del estudiante, para juzgar si es adecuado o no y tomar las medidas correspondientes" (D’Agostino de Cersósimo, 2007).

Por su parte el Art. 184 del Reglamento General de la Ley Orgánica de Educación Intercultural (2012) expresa: "La evaluación estudiantil es un proceso continuo de observación, valoración y registro de información que evidencia el logro de objetivos de aprendizaje de los estudiantes y que incluye sistemas de retroalimentación, dirigidos a mejorar la metodología de enseñanza y los resultados de aprendizaje".

Muchos son los autores que han aportado a la construcción del concepto de evaluación en el terreno educativo, sin embargo podemos apreciar que todos concurren en un proceso de recolección de información, que permita elaborar juicios de valor para tomar decisiones.

\section{Proceso de Evaluación de los Aprendizajes}

Quizá uno de los errores más frecuentes en el proceso educativo, ha sido ver la evaluación como una simple actividad que persigue asignar calificaciones a los estudiantes, para al final decidir o no su promoción al siguiente nivel de estudios. Ésta es una visión errónea de lo que significa evaluar, pero que indudablemente ha repercutido en el accionar de todos los actores involucrados en el proceso, fundamentalmente maestros y estudiantes.

Por otra parte, en más de una ocasión ha sucedido que cuando se pregunta al docente sobre los logros alcanzados por sus estudiantes o las falencias y dificultades que presentan, el docente simplemente recurre a su memoria, para indicar hechos o situaciones que han sucedido en el aula, pero no tiene evidencias documentadas de los resultados de aprendizaje de los alumnos, o en muchas veces confunde el informe de evaluación con el registro de calificaciones.

También es usual atribuir la exclusiva responsabilidad a los estudiantes por la falta de consecución de los objetivos educativos, si bien es cierto actualmente existen muchos factores que desvían la concentración del estudiantado, no es menos cierto que pocas veces el 
profesorado reflexiona sobre su propia práctica en el ánimo de tomar decisiones que permitan mejorar el proceso.

Las situaciones descritas anteriormente, en alguna medida se dan por no aplicar un proceso correcto y sistemático en lo que se refiere a la evaluación de los aprendizajes.

A nuestro criterio la evaluación, al constituirse en parte fundamental de la educación, implica un proceso muy serio que consta de varios pasos imprescindibles (Casellas \& Jorba, 1997; Díaz Barriga \& Hernández, 2002; Ministerio de Educación y Cultura, 2002).

En el caso de la educación matemática es importante plantear como se han de abordar las diferentes etapas del proceso evaluativo. Ver tabla 1.

\section{Tabla 1}

\section{Etapas del proceso de evaluación de los aprendizajes en la educación matemática}

1. Determinación del Dentro del proceso educativo se pueden evaluar muchos aspectos, como por objeto a evaluar ejemplo: los aprendizajes de los estudiantes, el desempeño docente, la pertinencia de los recursos utilizados, etc. Es importante que como primer paso se determine qué se desea evaluar. En el caso del área de matemáticas se puede evaluar: La capacidad de los estudiantes para traducir un problema común en lenguaje matemático, la comprensión de conceptos, la resolución de problemas, aplicación de los aprendizajes en nuevas situaciones. El docente al momento de determinar el objeto a evaluar, está especificando el punto al cual llegar en este complejo sendero que implica el proceso educacional.

2. Determinación de

Permiten tener un parámetro de referencia con los cuales el evaluador puede juzgar los criterios de evaluación. si se está o no alcanzando los objetivos planteados en el punto anterior. Por ejemplo si se desea evaluar la capacidad de los estudiantes para resolver problemas, algunos de los criterios pueden ser: a) Identificación de los principales datos que proporciona la información del problema, b) Representación adecuada del problema mediante un gráfico, c) Uso de un proceso lógico y coherente que permita resolver el problema, d) Llegar a la respuesta correcta, e) Explorar otras formas de solución, f) Comprobar los resultados, entre otros. Recordemos que la educación es un proceso integral, el cual pretende formar a los estudiantes no sólo en aspectos de conocimientos de la asignatura, sino también en la formación de sus valores como ser humano; por ello se pueden incluir ciertos tipos de criterios como: puntualidad, orden, presentación, etc. Es importante indicar que los criterios pueden ser determinados en conjunto con los estudiantes, decisión que enriquecerá el proceso; en el caso de que no sea así es importante que al menos se los dé a conocer con anterioridad.

3. Recolección de la Se trata de aplicar instrumentos de evaluación, como portafolios, rúbricas, información cuestionarios, pruebas, etc., que permitan recolectar información, acerca del desempeño de los estudiantes.

Una vez recogida la información se procederá a compararla con los criterios

4. Análisis de la información establecidos en el punto 2; es importante que esta etapa no se conciba como la etapa de la simple calificación y la asignación de una nota, sino más bien en un proceso que debe propender a generar información retro-alimentadora que sirva a docentes y estudiantes para reflexionar sobre su práctica.

5. Emisión de juicios Constituye un aspecto importante del proceso, es la etapa en la cual el evaluador emite un juicio de valor de naturaleza cualitativa acerca del desempeño que han alcanzado los estudiantes.

6. Toma de decisiones Todas las etapas anteriores conllevan al aspecto más importante de la evaluación, la toma de decisiones, las cuales deben estar siempre encaminadas a conseguir mejoras significativas en el proceso educacional. 
En el momento que se concluye el proceso y se llega a la última etapa de la toma de decisiones, el docente se enfrenta a decisiones de carácter pedagógico y otras de carácter social, las cuales se pueden comprender de mejor manera a partir de la conceptualización de las funciones que se presentan en el proceso evaluativo.

\section{Funciones de la Evaluación}

\section{Función Pedagógica}

"La función pedagógica tiene que ver directamente con la comprensión, regulación y mejora de la situación de enseñanza y aprendizaje" (Díaz Barriga \& Hernández, 2002). Como se manifestó en líneas anteriores uno de los objetivos principales de la evaluación educativa es mejorar el proceso enseñanza-aprendizaje, es por ello que la información que se recoja debe servir para reflexionar sobre la metodología utilizada por el docente, los recursos utilizados, la relación docente-estudiante, la relación de los estudiantes con sus pares, el proceso comunicativo, la planificación curricular, los intereses de los estudiantes, etc., aquella reflexión debe servir para tomar los ajustes necesarios en el proceso, los mismos que permitan tomar correcciones de manera oportuna sobre aquellos aspectos que están obstaculizando el proceso, así como fortalecer aquellos que están bien encaminados.

Es preciso considerar que la función pedagógica hecha una mirada no únicamente sobre los aprendizajes de los estudiantes, por el contrario trata de analizar todos los componentes del proceso y los aborda de forma integral.

\section{Función Social}

En el momento en que un docente o una institución certifica que los estudiantes han alcanzado los objetivos educativos correspondientes a cierto nivel, o que están aptos para insertarse en estudios posteriores como por ejemplo de bachillerato o de educación superior, o en el momento en que se certifica que están aptos para su inserción en el mundo laboral, se debe entender que la evaluación ha cumplido su función social.

Si bien es cierto, esta función es importante dentro del proceso, es fundamental saber identificarla y utilizarla en el momento adecuado, evitando que opaque el accionar de la función pedagógica.

Para Díaz Barriga \& Hernández (2002) es necesario ponerle límites a la función social ya que en muchas ocasiones ha competido con la función pedagógica y ha llegado a anularla, debido a una inadecuada interpretación de la misma por la idea que prevalece de ver la evaluación relacionada simplemente con la calificación y la acreditación.

\section{La Evaluación de los Aprendizajes Desde un Enfoque Constructivista}

Varios son los cambios que ha experimentado el currículo del Sistema Nacional de Educación en los últimos años. En 1996 se planteó la Reforma Curricular de la Educación General Básica, en 2010 la Actualización y Fortalecimiento Curricular de la Educación General 
Básica, en 2011 el Currículo para el Bachillerato General Unificado y en 2016 el Currículo de los Niveles de Educación Obligatoria. Para El Ministerio de Educación del Ecuador (MinEduc, 2016) el actual ajuste curricular parte de los diseños curriculares anteriores y toma en consideración la experiencia de los docentes presentando así una propuesta más abierta y más flexible. A través del documento de Actualización y Fortalecimiento Curricular de la Educación General Básica, el MinEduc (2010) expresa que las bases pedagógicas que sustentan la propuesta curricular son la Pedagogía Crítica, que ubica al estudiantado como protagonista principal del aprendizaje, dentro de diferentes estructuras metodológicas, con predominio de las vías cognitivistas y constructivistas.

Sin embargo consideramos que falta mucho por profundizar en lo que se refiere a la evaluación de los aprendizajes desde esta perspectiva, por tal razón presentamos las principales características de la evaluación, desde una visión comparativa de un modelo pedagógico tradicional con otro constructivista, que sirvan de base para realizar un análisis de los tipos de evaluación, así como para proponer diferentes técnicas e instrumentos evaluativos a ser aplicados en la educación matemática. Ver tabla 2.

\section{Tabla 2}

\section{Características de la Evaluación desde la perspectiva de dos modelos pedagógicos}

\section{Modelo tradicional}

- Predomina el conocimiento memorístico de hechos, datos y conceptos.

- Se enfatiza los productos y no los procesos, de los productos generalmente se resalta la vertiente negativa

- Evaluación netamente cuantitativa, pues el examen de lápiz y papel es el principal instrumento de evaluación.

- Se prioriza demasiado la función social de la educación, de manera particular la evaluación sumativa y acreditativa.

- Generalmente se evalúa el aprendizaje de los alumnos y no la enseñanza.

- $\quad$ El docente es casi siempre quien define la situación evaluativa, a veces de forma autoritaria, sin dar a conocer a los alumnos el por qué y el para qué de la evaluación.

- Para la evaluación se seleccionan los ejercicios que casi nunca se revisan en clase, pensando en que la transferencia o generalización de saberes ocurre de forma espontánea.

- Evalúa el aprendizaje descontextualizado, planteando situaciones artificiales, que en ocasiones generan desinterés y desmotivación en los estudiantes.
Modelo constructivista

- Pone énfasis en la evaluación de los procesos de aprendizaje.

- Evalúa la significatividad de los aprendizajes. De este modo resulta deseable que el aprendiz logre, junto con ciertos aprendizajes significativos, un conocimiento condicional que le permita saber qué sabe, cómo lo sabe, y en qué y para qué contextos le puede resultar útil eso que sabe.

- Evalúa la funcionalidad de los aprendizajes, es decir que los estudiantes puedan utilizar sus aprendizajes para resolver problemas o para conectarlos con aprendizajes nuevos.

- Propicia un aprendizaje autorregulado por parte de los estudiantes

- Evalúa y regula la enseñanza, da una fuerte importancia a la función pedagógica de la evaluación, reflexiona permanentemente sobre los procesos de enseñanza, para tomar oportunamente medidas correctivas, de ser el caso.

- Evalúa aprendizajes contextualizados, obviamente generando aprendizajes apegados a la realidad en el proceso de enseñanza. Se trata de que el estudiante utilice lo aprendido para la formulación y solución de problemas reales.

- Propicia un proceso de autoevaluación del alumno, lo cual ayuda en el proceso de autorregulación de sus aprendizajes. Persigue que el alumno entienda el por qué y el para qué de la evaluación y haga suyos los objetivos educacionales.

- Fomenta la coherencia entre las situaciones de aprendizaje y las situaciones de evaluación. 
Fuente: (Díaz Barriga \& Hernández, 2002)

\section{Tipos de Evaluación}

En la diversa literatura referente al tema, varias son las propuestas para clasificar los diferentes tipos de evaluación, la que será desarrollada aquí intenta guardar coherencia con lo que plantea el marco legal del sistema educativo ecuatoriano y los documentos curriculares.

\section{Evaluación Diagnóstica}

Tiene como principal objetivo recoger información que permita al docente marcar un punto de partida para emprender un proceso de aprendizaje con sus estudiantes, ésta se la puede utilizar al inicio de un ciclo escolar, un módulo educativo, un bloque curricular, un quimestre, etc.

Por otra parte, busca que el docente mediante la aplicación de diferentes técnicas e instrumentos pueda identificar plenamente cuáles son los conocimientos previos de sus estudiantes, sus intereses, sus expectativas en el proceso, etc.

"Cuando la información que se obtiene a partir de dicha modalidad de evaluación [diagnóstica] hace referencia a un colectivo (grupo-clase), se denomina prognosis, y cuando es diferenciada (de cada alumno/a) se llama diagnosis" (Jorba \& Sanmartí, 1993).

Incluso dentro de la evaluación diagnóstica se pueden considerar dos subcategorías, la evaluación inicial y la evaluación puntual. La evaluación inicial, se la realiza exclusivamente antes de un proceso o ciclo educativo amplio, busca identificar si los estudiantes poseen los prerrequisitos para enfrentar de manera exitosa el ciclo de estudios. La evaluación puntual tiene como finalidad identificar y utilizar continuamente los conocimientos previos de los alumnos, se realiza de forma más permanente al iniciar un tema o una clase. (Díaz Barriga \& Hernández, 2002).

Luego de haber obtenido la información y haberla analizado, se procede a tomar decisiones tales como arrancar el programa de estudio sin ningún inconveniente o realizar unos pequeños ajustes a la programación curricular, que permita a los estudiantes acoplarse al período de estudios.

\section{Evaluación Formativa}

Quizá sea la más importante en todo el proceso, está muy relacionada con la función pedagógica de la evaluación, pues persigue reflexionar tanto sobre los procesos de aprendizaje y de enseñanza, ya que le da mayor importancia a los procesos que a los resultados. Díaz Barriga y Hernández (2002) expresan que "no importan tanto valorar los resultados, sino comprender el proceso, supervisarlo e identificar los posibles obstáculos o fallas que pudiera haber en el mismo, y en qué medida es posible remediarlos con nuevas adaptaciones didácticas in situ."

Es útil para los estudiantes, porque con la orientación permanente del docente pueden superar oportunamente las dificultades que se presentaren, también es útil para el docente, ya que 
le proporciona información sobre cómo mejorar su práctica profesional. Según varios autores, se distinguen tres formas de regulación formativa: interactiva, retroactiva y proactiva.

Allá (como se citó en Jorba \& Sanmartí, 1993) indica que la regulación interactiva está integrada en la situación de aprendizaje, pues a través del material didáctico se produce esa interacción del estudiante con sus compañeros y con el docente. Por su parte, la regulación retroactiva programa situaciones de refuerzo, y aspira ayudar a los estudiantes a superar las dificultades detectadas en la evaluación. Finalmente la regulación proactiva prevé actividades futuras que buscan afianzar los aprendizajes de los estudiantes.

Dentro de la evaluación formativa está la evaluación formadora, esta última está orientada a promover en el alumno la capacidad de regular sus propios procesos de aprendizaje, lo cual implica que incluso sea el propio alumno que en determinada medida evalúe sus propios aprendizajes. Surgen entonces tres tipos de evaluación, que utilizadas correctamente fortalecen la evaluación formativa:

La autoevaluación: se trata de la evaluación del alumno acerca de su propio trabajo. La coevaluación, el alumno realiza la evaluación de su trabajo con el acompañamiento del docente y la evaluación mutua, que es la realizan los estudiantes a las producciones de sus pares. (Díaz Barriga \& Hernández, 2002).

\section{Evaluación Sumativa}

Es aquella que se realiza al final de una unidad, de un bloque, de un quimestre, etc., en ella predomina la función social de la evaluación, ya que persigue objetivos de calificación y acreditación y de esta manera verificar si los objetivos propuestos al inicio del período fueron o no alcanzados.

Al ser un tipo de evaluación muy relacionada con lo que es calificación y certificación, se centra mucho en la evaluación de los productos y no de los procesos. Si bien es importante evaluar los productos, se debe tener cuidado de que la práctica evaluativa del profesorado no quede reducida al simple hecho de asignar calificaciones, si aquello ocurre no se estaría entendiendo la evaluación como un proceso integral y por consiguiente se perdería la oportunidad de tomar decisiones muy importantes en el proceso como consecuencia de la información que surge en el mismo. Ver Figura 1. 


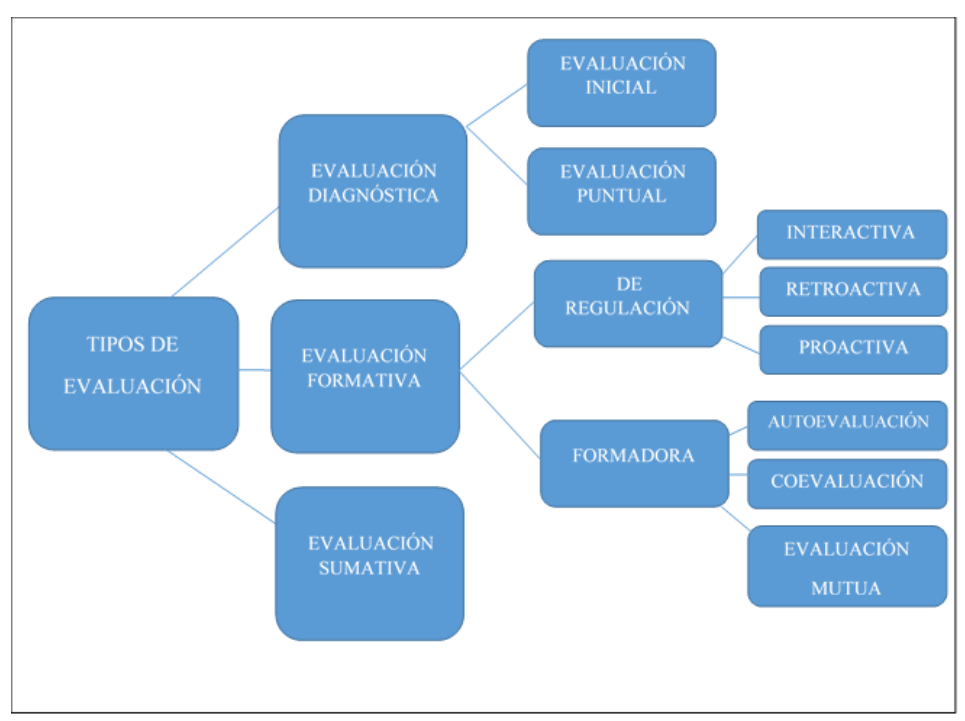

\section{Técnicas e Instrumentos de Evaluación}

Como se ha mencionado, son muchos los aspectos que se pueden evaluar en el proceso educativo, lo cual significa que se pueden seleccionar muchos objetos a evaluar -los recursos, el proceso de aprendizaje de los estudiantes, el desempeño del docente, entre otros-, indudablemente que dependiendo de qué se desee evaluar se escogerá el mecanismo de cómo hacerlo, es decir se decidirá de qué manera y mediante qué recursos se puede obtener la información, que luego tendrá que ser tratada adecuadamente.

En nuestro sistema de educación, cuando se habla de evaluación del aprendizaje, tanto docentes como estudiantes inmediatamente asocian el proceso con la aplicación de pruebas y exámenes, pues consideramos que si bien es cierto la prueba permite obtener información sobre el aprendizaje de los estudiantes, no es menos cierto que no es la única manera de hacerlo.

Debemos considerar que la utilización de recursos para la obtención de información, es altamente dependiente de varios factores como: asignatura a evaluarse, tiempo disponible, delimitación del objeto a evaluar, es decir se quiere evaluar cálculos matemáticos, resolución de problemas o comunicación de las ideas matemáticas, etc.

El lector podrá advertir que una vez seleccionados los objetos de evaluación, debe considerar cómo y con qué recursos puede recabar la información que necesita. "De la pertinencia de las técnicas seleccionadas y de la calidad de los instrumentos que se construyan, se derivará la calidad de la información obtenida; de lo cual dependen los juicios y las decisiones que posteriormente se tomen" (Ministerio de Educación y Cultura, 2002).

Según el diccionario de la Real Academia Española (RAE, 2017), por técnica se entiende "el conjunto de procedimientos y recursos de que se sirve una ciencia o un arte", en tanto que instrumento se define como "aquello que sirve de medio para hacer algo o conseguir un fin".

Por consiguiente en nuestro contexto educativo entendemos a las técnicas de evaluación, como los procedimientos y actividades que permiten efectivizar el proceso. 
En tanto que los instrumentos son entendidos como los medios que facilitan al docente recoger información del objeto que pretende ser evaluado.

Un aspecto esencial de todo el proceso es que el docente sepa decidir acertadamente qué técnicas e instrumentos utilizar dependiendo de lo que pretenda evaluar, esta habilidad la irá adquiriendo por medio de su experiencia profesional por un lado, pero también por el conocimiento teórico que tenga al respecto.

A continuación ponemos a consideración varias técnicas con sus respectivos instrumentos que pueden ser utilizados para evaluar los aprendizajes en la educación matemática.

\section{La Observación}

De forma permanente el docente está observando el accionar de sus estudiantes, observa por ejemplo la actitud y predisposición que tienen para un trabajo en grupo, observa qué temas son los que más despiertan su interés así como los que menos les interesa, observa las relaciones interpersonales existentes entre ellos, observa incluso sus expresiones gestuales y corporales durante la clase, sin embargo la mayor parte de docentes no registra esta valiosa información ocasionando que la misma pase desapercibida.

Para que el proceso de observación de resultados exitosos, se recomienda seguir ciertas pautas: a) Definir los objetivos de la observación: es decir establecer que es lo que se ha de evaluar. b) Especificar los datos a obtener: qué es lo que se pretende observar, como por ejemplo la comunicación que entablan los alumnos entre sí. c) Elaborar o seleccionar los instrumentos adecuados: se refiere a los medios que me permitirán recoger la información. d) Registrar lo observado: es necesario hacerlo de forma inmediata para evitar que los datos sean olvidados. e) Contrastar la información: se puede recurrir a otros observadores y contrastar los datos medidos. (MEC, 2002).

Los instrumentos que pueden ser utilizados por los docentes para esta técnica son:

\section{Registro Anecdótico}

Es un instrumento en el que "se describen por escrito episodios, secuencias, etcétera, que se consideran importantes para evaluar lo que interesa en un alumno o en un grupo de alumnos" (Díaz Barriga \& Hernández, 2002).

Cuando se hace uso de este instrumento, es necesario que el docente registre imparcialmente los hechos que observa, evitando anticipar tempranamente juicios de valor que podrían distorsionar el proceso.

Por asuntos de carácter práctico proponemos que se designe un cuaderno para llevar los registros anecdóticos de los alumnos y que cada página de éste contenga la información de un estudiante, el formato que constaría en cada página se muestra en la tabla 3, también proponemos un ejemplo de cómo llenar cada parte del mismo. Vale indicar que en el formato se puede agregar la foto del estudiante. 
Por otra parte al final del quimestre se puede realizar un resumen de lo observado durante este periodo, este es un instrumento clave para la realización de un informe de evaluación quimestral.

Tabla 3. Registro anecdótico

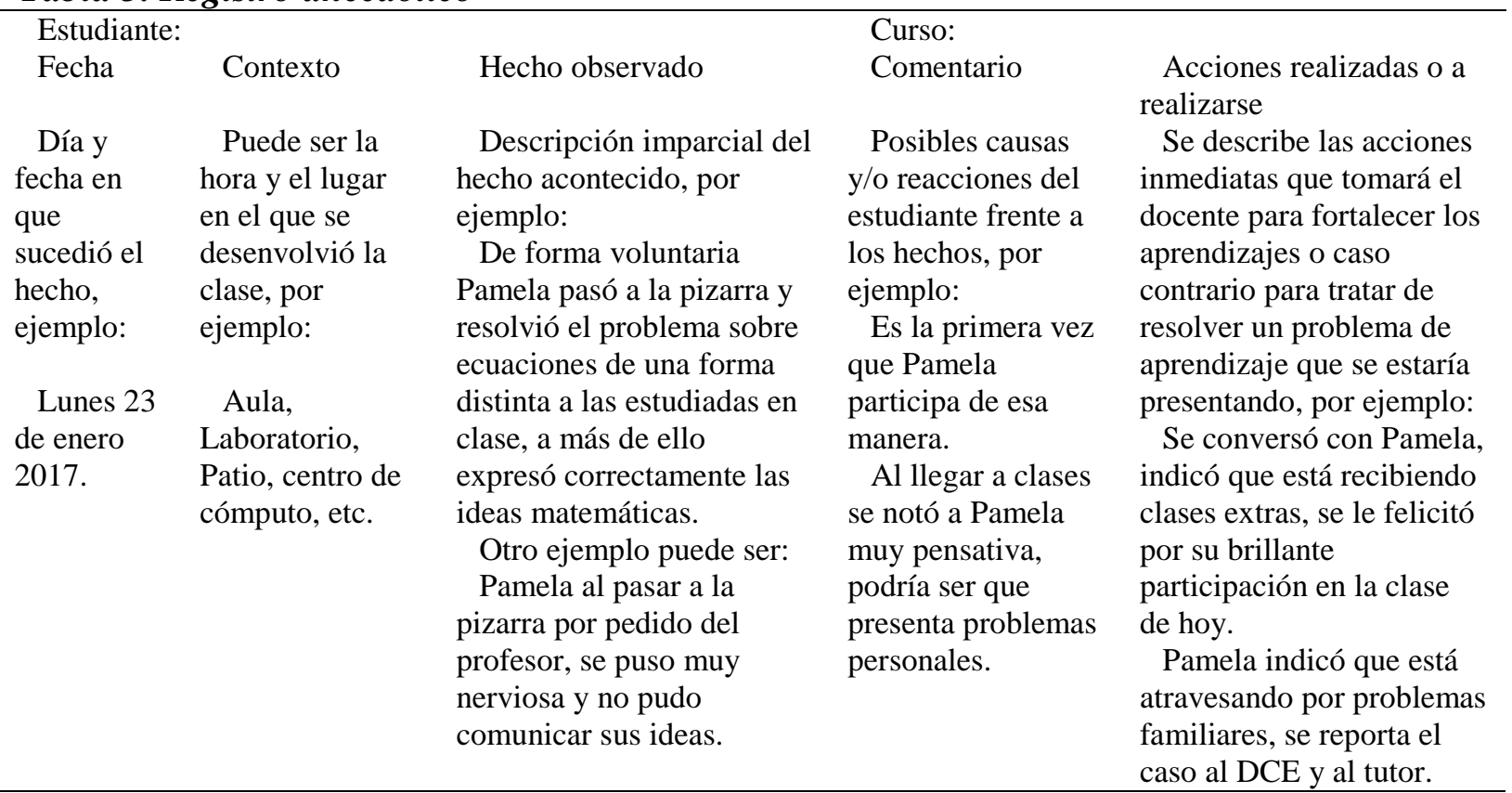

Nota: Adaptado de: (MEC, 2002).

\section{Lista de Cotejo}

Es un instrumento que permite evidenciar el nivel de desarrollo de las destrezas con criterios de desempeño por parte del estudiante, el docente puede hacer uso de este instrumento cuando propone una tarea ya sea grupal o individual y recorre por los puestos de trabajo de cada estudiante o de cada grupo observando el actuar de los mismos, puede servir también cuando pide a sus estudiantes que expongan determinado trabajo que ha sido preparado previamente. Para su elaboración, se establecen las siguientes recomendaciones:

Especificar la destreza o actuación a ser observada, especificar los rasgos centrales de la actuación, ordenar los elementos enumerados y ordenarlos en categorías si es necesario, diseñar el formato del instrumento. (MEC, 2002).

A continuación proponemos un ejemplo de una lista de cotejo que puede servir para evaluar destrezas con criterios de desempeño del bloque de Estadística y probabilidad correspondiente al subnivel superior de Educación General Básica. Ver tabla 4.

Tabla 4

Lista de cotejo para evaluar destrezas con criterio de desempeño del bloque de Estadística

Destreza:

Grupo $\mathrm{N}^{\circ}$ Estudiantes

Saúl Ana Rita Paúl Eva




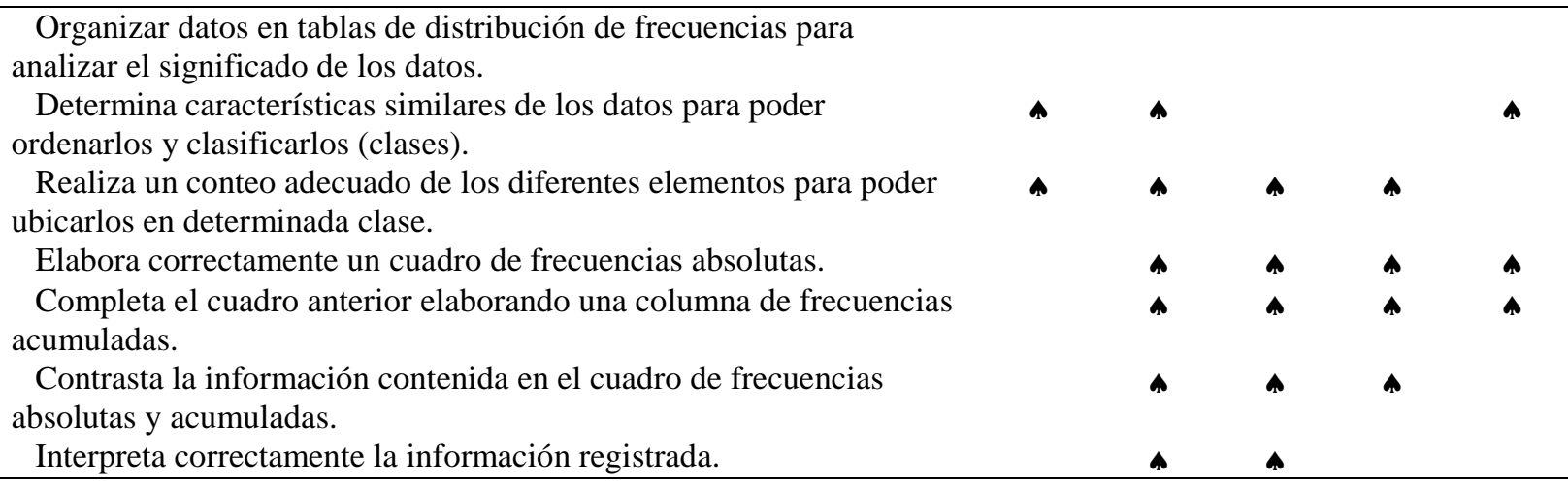

Es preciso que para el uso de este instrumento se plantee a los estudiantes una actividad que permita evidenciar que han logrado el desarrollo de la Destreza con Criterio de Desempeño, es recomendable además que las actividades sean contextualizadas y haciendo uso del material del entorno, se puede trabajar por ejemplo con datos como: Estaturas de los estudiantes, edades, número de hermanos que tienen, número de horas al día o a la semana que dedican a sus estudios, número de horas al día o a la semana que dedican a las redes sociales, permitiendo incluso al docente hacer una reflexión con la información proporcionada.

\section{Escala de Valoración Numérica}

La escala de valoración numérica permite contestar mediante una escala numérica la siguiente pregunta ¿Qué tanto alcanzaron los estudiantes determinada destreza con criterio de desempeño?, puede presentar una ventaja respecto a la lista de cotejo ya que proporciona información más precisa acerca de lo observado, al igual que el instrumento anterior se establecen algunas recomendaciones para su elaboración.

Especificar la DCD a ser observada, especificar las instrucciones para su uso, se recomienda un número par de grados de valoración con el objeto de evitar que el valor quede siempre en el centro y aquello no proporcione una información significativa, se puede empezar la escala desde el grado 0 o 1 , dependiendo de las necesidades del docente, se puede emplear la primera opción en el caso de que se quiera dejar muy clara la ausencia de la destreza o comportamiento, por otra parte es recomendable que la escala tenga un máximo de 10 grados de valoración. (MEC, 2002).

Al igual que en el punto anterior, proponemos un ejemplo, en esta ocasión concerniente al bloque de Geometría y Medida del subnivel superior de Educación General Básica. Ver tabla 5. 


\section{Tabla 5 \\ Escala de valoración numérica para evaluar destrezas con criterios de desempeño del bloque de Geometría y Medida}

\footnotetext{
Módulo:

Destreza con criterio de desempeño: Clasificar y construir triángulos, utilizando regla y compás, bajo condiciones de ciertas medidas de lados y/o ángulos.
}

Instrucciones: Valore el grado de adquisición de la destreza encerrando en un círculo el valor que corresponde, considerando que 1 es lo mínimo y 6 lo máximo.

NÓMINA DE ESTUDIANTES

\begin{tabular}{cccccc}
$\begin{array}{l}\text { VALORACIÓN } \\
1\end{array} 2$ & 3 & 4 & 5 & 6 \\
1 & 2 & 3 & 4 & 5 & 6 \\
1 & 2 & 3 & 4 & 5 & 6 \\
1 & 2 & 3 & 4 & 5 & 6 \\
\hline
\end{tabular}

Nota: Adaptado de: (MEC, 2002)

Una de las exigencias actuales del Ministerio de Educación consiste en que el docente recoja todas las evidencias acerca del progreso de los aprendizajes de sus estudiantes.

En este contexto la escala de valoración numérica puede constituirse en un poderoso instrumento que permita registrar tal información, si bien es cierto puede demandar un gran trabajo del docente para su elaboración y aplicación, es importante señalar que brinda una excelente oportunidad para la auto-evaluación y co-evaluación de los estudiantes. Estamos conscientes de que estos procesos aplicados por parte de los estudiantes pueden proporcionar información subjetiva, pero también estamos conscientes de que el profesorado debe aplicarlos y guiarlos adecuadamente, reflexionando constantemente sobre los mismos sólo de esta manera se logrará que el estudiante se involucre con su propio proceso de evaluación y se pueda generar una verdadera información retroalimentadora.

En el caso de que el docente decida utilizar este instrumento para la autoevaluación de sus estudiantes, se recomienda que el docente después de cada destreza de haber trabajado pida a los estudiantes que elaboren su propia tabla y la coloquen en su cuaderno de trabajo, en este caso la escala de valoración tendrá el encabezado y tan solo una fila correspondiente al nombre del estudiante.

Si se utiliza una metodología de trabajo grupal se puede pedir a cada grupo que elabore una tabla, la cual tendrá el número de filas de acuerdo al número de integrantes del grupo, en la cual cada miembro evaluará a uno de sus compañeros.

Si bien es cierto se presentan varias opciones, es el docente quién debe elegir cual es la más adecuada para su contexto.

\section{Los Trabajos Realizados por los Estudiantes}

Generalmente los docentes suelen enviar a sus estudiantes tareas sobre los aprendizajes trabajados en el aula, estas pueden ser de diversos tipos como por ejemplo: trabajos de investigación, resolución de ejercicios y/o problemas, elaboración de mapas conceptuales, etc. 
La información que pueden otorgar esas producciones de los estudiantes es muy enriquecedora para el proceso.

Vale también considerar que el área de matemáticas tradicionalmente ha sido juzgada por la cantidad de ejercicios que se envía a los estudiantes a sus hogares, si bien es cierto en algunos temas de la matemática quizá sea necesario hacerlo, vale reflexionar que actualmente se trata de ver la enseñanza de la matemática con un nuevo enfoque, no enmarcada tan solo en la repetición de procesos y algoritmos mecánicos, que en algunos casos pueden resultar descontextualizados y sin sentido para los aprendices, sino más bien en un proceso reflexivo que fomente el razonamiento lógico y crítico en los estudiantes. En ciertas ocasiones un solo problema bien planteado y contextualizado puede ser más enriquecedor que el desarrollo de muchos ejercicios aislados. Al respecto (Díaz Barriga \& Hernández, 2002) manifiestan que "un trabajo o ejercicio bien seleccionado, informativo y motivante provoca mayores dividendos en el aprendizaje de los alumnos y en la evaluación del profesor sobre sus progresos, que cualquier otro que se repita incesantemente y que no tenga sentido ni valor funcional".

Es fundamental que el profesor revise permanentemente las tareas enviadas a sus estudiantes, pues esta información es un importante recurso para el desarrollo de la evaluación formativa y propicia un excelente escenario para retroalimentar los aprendizajes. Es una buena práctica del docente de matemáticas preguntar a sus estudiantes al inicio de su clase que inconvenientes tuvieron en la realización de la tarea, y a partir de sus ideas ir encontrando las mejores soluciones, no se trata que el profesor sea quien siempre resuelve los problemas y los estudiantes solo observan pasivamente, recordemos que el constructivismo ubica al docente en un lugar de facilitador de los aprendizajes, siendo los estudiantes quienes son los principales actores del proceso de construcción de su aprendizaje.

Vale indicar que la producción de los estudiantes no se da únicamente mediante las tareas que realizan en sus hogares, es necesario también que el docente destine un tiempo dentro de sus horas de clase para plantear actividades a sus estudiantes y monitorear el avance de las mismas.

En este punto también se puede utilizar mucho la coevaluación y autoevaluación. Quizás estos son los momentos más oportunos en los que se puede ir enseñando a los alumnos cómo evaluar sus procesos y productos -uso de la coevaluación primero y, posteriormente, de la autoevaluación-. Asimismo, conviene utilizar estrategias de evaluación mutua entre compañeros, para que estos comparen sus trabajos con una serie de criterios bien definidos y discutan abiertamente y respetuosamente, guiados por el enseñante, sobre sus avances logrados. (Díaz Barriga \& Hernández, 2002).

\section{El Portafolio}

Un portafolio es un "conjunto intencionado de trabajos que muestran los esfuerzos, progresos y logros de los estudiantes en una o más de una de las áreas curriculares. El sentido de esta colección es ilustrar el progreso a lo largo del tiempo” (MEC 2002). 
Es una técnica que brinda información tanto sobre los avances de los estudiantes, pero también puede brindar información sobre las acciones de enseñanza, en este sentido la evaluación se apega a su función formativa.

A continuación presentamos una propuesta de portafolio para ser utilizada por los docentes del área de matemáticas. El portafolio puede ser presentado en una carpeta, pero recomendamos que tenga los siguientes componentes:

1. Una portada, cuyo formato puede ser planteado por el docente o se puede dejar a criterio de la iniciativa y creatividad del estudiante.

2. Un texto corto, elaborado por el estudiante, en el que incluya sus percepciones de la asignatura, sus fortalezas y sus temores, así como las expectativas que tiene para el presente quimestre.

3. Los enunciados de unos cinco ejercicios o problemas seleccionados por el estudiante y que a su criterio posean un gran nivel de dificultad.

4. Por cada bloque curricular la realización de un ejercicio seleccionado por el estudiante, con el criterio de que no tiene ningún inconveniente para resolverlo.

5. El trabajo menos satisfactorio durante el quimestre.

6. El mejor trabajo durante el quimestre

7. El avance de la resolución de los ejercicios o problemas seleccionados en el punto 2, acompañado cada uno de una reflexión sobre su proceso de aprendizaje.

8. La resolución de un ejercicio que le parezca que le puede ser muy útil en su vida cotidiana.

9. Una autoevaluación a su desempeño durante cada quimestre.

10. Una breve evaluación al desempeño del docente. Se trata de que el estudiante en un párrafo exprese las fortalezas del docente, así como sus debilidades, debe estar en una hoja aparte y sin nombre. Este punto se convierte en aspecto central del proceso enseñanza aprendizaje, se trata de que el docente reciba a sus estudiantes está información, la procese y la utilice para mejorar su práctica pedagógica.

También proponemos el respectivo instrumento de evaluación para el mismo: 
1. Entregó todos los puntos del portafolio (9 puntos). Falta:

2. Existe una buena presentación del portafolio (1 punto).

3. Aplica procedimientos correctos para solucionar los problemas matemáticos ( 1 punto por cada problema).

4. Son correctas las respuestas de los ejercicios resueltos ( 1 punto por cada ejercicio).

5. Hay evidencia de su progreso en su proceso de aprendizaje (10 puntos)

6. La autoevaluación evidencia un proceso de reflexión constante y serio ( 5 puntos)

TOTAL:
Puntuación

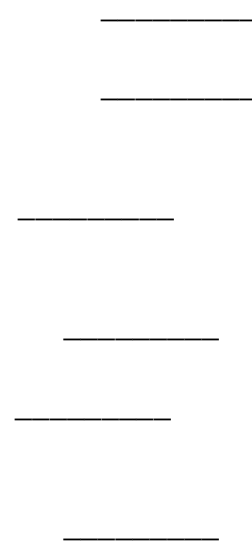

\section{COMENTARIOS:}

\section{FIRMA DEL PROFESOR}

Figura 2. Evaluación del Portafolio.

Dejamos a criterio del docente el uso de esta técnica, la misma puede ser utilizada ya sea para la evaluación de un período quimestral o ya sea para la evaluación de un año lectivo.

Finalmente consideramos que muchas son las técnicas que se pueden utilizar para evaluar los aprendizajes en la asignatura de Matemáticas, todo dependerá del conocimiento y de la experiencia del profesorado para poder utilizar los distintos instrumentos en la particularidad de los contextos que se presentan en el proceso educativo.

\section{Bibliografía}

Casanova, M. A. (1998). La evaluación educativa. (La Muralla, Ed.).

Casellas, E., \& Jorba, J. (1997). Estrategias y técnicas para la gestión social del aula: La regulación y autorregulación de los aprendizajes. (Síntesis, Ed.) (1era ed.). Madrid.

D’Agostino de Cersósimo, G. (2007). Aspectos teóricos de la evaluación educacional. (Editorial Universidad Estatal a Distancia, Ed.) (Primera). San José.

Díaz Barriga, F., \& Hernández, G. (2002). Estrategias docentes para un aprendizaje significativo: una interpretación constructivista. (McGraw-Hill, Ed.) (2a ed.). México, D.F.

Jorba, J., \& Sanmartí, N. (1993). La función pedagógica de la evaluación. Aula de Innovación Educativa, 20. Retrieved from http://www.grao.com/revistas/aula/020-la-evaluacion-enel-proceso-de-ensenanza-aprendizaje--secuenciacion-de-los-contenidos-de-lengua/la- 
funcion-pedagogica-de-la-evaluacion

Ministerio de Educación del Ecuador. (2010). Actualización y Fortalecimeinto Curricular de la Educación General Básica. (M. Guitarra, Ed.) (1era ed.). Quito.

Ministerio de Educación del Ecuador. (2016). Currículo de los Niveles de Educación Obligatoria. Retrieved from http://educacion.gob.ec/wpcontent/uploads/downloads/2016/08/Curriculov2.pdf

Ministerio de Educación y Cultura. (2002). Evaluación de los aprendizajes. (Orión, Ed.) (1era ed.). Quito.

Quinquer, D. (1999). Modelos y enfoques sobre la evaluación : el modelo comunicativo. Aula de Innovación Educativa, 80, 54-57.

Real academia española. (2017). Real Academia Española. Retrieved from http://dle.rae.es/?id=ZIkyMDs

Reglamento General a la ley Orgánica de Educación Intercultural, Pub. L. No. 1241 (2012). Ecuador. 